\title{
MicroRNA sponge production using PCR-based concatemerization of short DNA oligonucleotides
}

\begin{abstract}
Micro RNAs (mi Rs) are small, non coding RNAs encoded within our genome that regulate gene expression by silencing messenger RNA (mRNA) transcripts. Since being discovered in humans in 2001, much has been learned concerning the many cellular activities that mi Rs can affect. Mi Rs have been implicated in almost every cellular activity, including cell fate determination, stress response, metabolism, apoptosis, and carcinogenesis. Importantly, alterations in mi R activity due to mutation or mis expression have been repeatedly shown to result in tumorigenesis and disease. However, because of their relatively recent discovery, therapeutic tools to suppress mi Rs continue to be developed. One extremely promising way to inhibit mi Rs is to use mi R sponges which consist of $\sim 15 \mathrm{mi}$ R "target" sites that can specifically bind and inactivate particular mi Rs. Unfortunately, mi $\mathrm{R}$ sponge production has proven to be problematic thus far as current production methods involve either costly commercial synthesis or low throughput ligation-based cloning. However, we have recently developed a novel, PCR-based method that allows sponge production in a rapid, high throughput manner. In all, PCR amplification, cloning, sequence confirmation and large scale production are readily achievable in less than two weeks and at a significantly lower cost than current methods.
\end{abstract}

Volume 2 Issue 2 - 2015

\author{
Sara-Elizabeth Cardin,' Tabitha J Perry,' \\ Caroline J Polska,' Laura Valverde,' Alyson \\ N Askew,' Dillon G Patterson,' Glen M \\ Borchert $^{1,2}$ \\ 'Department of Biology, University of South Alabama, USA \\ ${ }^{2}$ Department of Pharmacology, University of South Alabama, \\ USA
}

Correspondence: Glen M Borchert, Department of Biology \& Pharmacology, Assistant Professor of Biology \& Pharmacology, University of South Alabama, Alabama, USA, Tel (25I) 46073I0, Fax (25I) 4I48220, Email borchert@southalabama.edu

Received: April 27, 2015 | Published: May 22, 2015

Keywords: micro rna, mi rna, mi r, mi r inhibitors, mi r sponge, per

Abbreviations: Bp, base pair; CE RNA, competing endogenous rnas; Circ RNA, circular rna; Ci RS, circular rna sponges; LINC RNA, long intervening non coding rnas; LNA, locked nucleic acid; Mir, micro rna; MRNA, messenger rna; NT, nucleotide; PCR, polymerase chain reaction; Pre-Mi R, precursor micro rna; Pri-Mir, primary micro rna; RISC, rna induced silencing complex; SIRNA, small interfering rna; UTR, un translated region

\section{Introduction}

Micro RNAs (mi Rs) are small, $\sim 20$ nucleotide (n t) non-coding RNAs that act as regulators of gene networks. Since being discovered in Caenorhabditis elegans in 1993, studies have identified over 2,500 unique human mi Rs and over 35,000 mature mi $\mathrm{R}$ products across species. Mi Rs are widespread in higher eukaryotes and similar in function to small interfering RNAs (si RNAs), in that they silence specific messenger RNAs (mRNAs). Generation of mi $\mathrm{R}$ from endogenous hairpin-shaped transcripts occurs in two main stages: within the nucleus and without. ${ }^{1}$ First, the mi R gene is transcribed within the nucleus by either RNA Polymerase II or III producing a long primary molecules, called a pri-mi $\mathrm{R}$ typically composed of several thousand nts. ${ }^{2}$ The pri-mi $\mathrm{R}$ is then cut by Drosha, yielding a $\sim 70 \mathrm{nt}$ long precursor stem loops (pre-mi R). Next, the pre-miR hairpin is exported to the cytoplasm by exportin-5, where Dicer cuts it down to $\mathrm{a} \sim 22 \mathrm{nt}$ duplex. One strand is degraded and the other is retained as the mature mi R that associates with the RNA induced silencing complex (RISC) ${ }^{1,3}$ From there, the complementarity between the 6-8nt 'seed' region of the mi $\mathrm{R}$ and the 'seed match' region on the mRNA determines the fate of the mRNA. Importantly, mi R binding does not have to be perfect in order to have an effect. When a mi R seed binds to the mRNA seed match, it can result in one of two things: mRNA cleavage in the event of perfect full-length mi R complementarity, or translational repression if the match is imperfect. Importantly, a single mi R can influence the activity of numerous mRNAs, an ability which in turn allows mi Rs to form intricate regulatory networks. ${ }^{5}$

Altered activities of several mi Rs due to mutation or mis expression have now been shown to result in disease onset and/or tumorigenesis. ${ }^{6-9}$ While miR mis regulation is implicated in numerous malignancies, therapeutic tools for mi $\mathrm{R}$ suppression have only recently been developed. ${ }^{6}$ Commercially available mi $\mathrm{R}$ inhibitors fall into three major categories: antagomirs, ${ }^{10}$ synthesized sponges, ${ }^{11}$ or antisense inhibitors. ${ }^{12}$ Antagomirs, better known as LNAs (locked nucleic acids) or anti-mi Rs, are chemically modified nucleic acids that strongly bind a target mi $\mathrm{R}$ by traditional complementary base pairing. The thermodynamic stability of LNA binding keeps them tightly associated with their targets, but also allows for imperfect base pairing at high temperatures. Unfortunately, LNAs carry the risk of off-target effects ${ }^{13}$ making them less desirable candidates for therapeutic endeavors despite their demonstrated utility as a tool for basic research. ${ }^{11}$ In contrast, antisense inhibitors (such as Ambion's mirVana) contain an unmodified RNA strand (which binds targets with normal affinity) and a chemically modified strand that cannot associate with RISC. Although this class of inhibitors is not hindered by the potential toxicity associated with LNAs, these antisense inhibitors can still prove problematic for many researchers in terms of the cost associated with their production. ${ }^{12}$ Significantly different in design than either of the synthetic inhibitors, the third type of mi $\mathrm{R}$ inhibitor currently employed is the mi R sponge. Mi R sponges are RNA transcripts with repeated binding sites forming a concatemer of targets for a specific mi R (Figure 1). Sponges can be constructed with repeats of any sequence, but sponges with central mismatches, called a central bulge, have been shown to be more effective at inhibiting mi $\mathrm{R}$ function than perfectly complementary sponges. ${ }^{11}$ To date, sponges have been synthesized by either commercial synthesis or T4 ligation reactions. While more cost effective than commercial synthesis, due 
to the linear nature of their reaction mechanism, T4 ligation reactions are notoriously low throughput and typically require large amounts of starting material to produce relatively low product yields. ${ }^{14}$

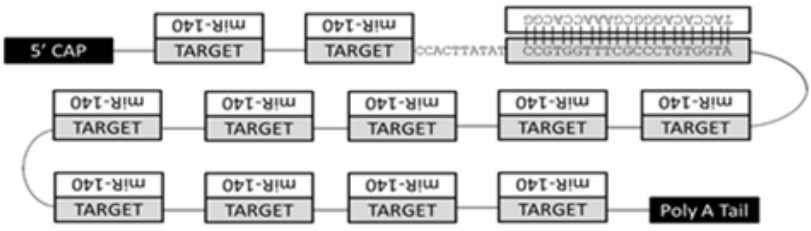

Figure I MicroRNA target binding: Cartoon illustrating a miR-|40 mi RNA sponge. Expressed from a eukaryotic promoter, sponges consist of capped, polyadenylated mRNAs baring multiple mi RNA "Target" sites. In all, twelve target sites are illustrated base paired to complementary miR-I40 mi RNAs.

Excitingly, we have developed an entirely novel, rapid, PCRbased methodology that greatly facilitates sponge production. The concatemerizing PCR technique we present here represents a costeffective and practical method for mi R sponge production. Instead of time-consuming ligation reactions that produce little overall yield, concatemerizing PCR utilizes amplification steps and exponential growth to quickly generate large quantities of sponge amplicons that consist of an easily user-defined number of mi $\mathrm{R}$ binding sites. Importantly, as prominent roles for mi Rs in disease development and progression continue to be described ${ }^{7-9,15}$ knocking down mi Rs in the laboratory and clinic has become an increasingly significant endeavor, and the method of sponge production described here constitutes an innovative, inexpensive, high yield means of mi R inhibition.

\section{Materials and methods}

\section{Primer design}

Approximately 40 nucleotide forward primers were constructed with GGTGAATATA at both the 5' and 3' ends flanking an internal sequence to be repeated and concatemerized. Reverse primers were ordered exactly as the reverse complement of the forward primer allowing concatemerization. Each sponge was concatemerized with the following primers:

\section{miR I 40 sponge:}

GGTGAATATATACCACAGGGCGAAACCACGGGGTGAATATA TATATTCACCCCGTGGTTTCGCCCTGTGGTATATATTCACC

SPG1:GGTGAATATATTAATACGTGACCTATTAATGGTGAATATA, TATATTCACCATTAATAGGTCACGTATTAATATATTCACC

SPG2:GGTGAATATATAGCTTATCAGACTGATGTTGAGGTGAATATA, TATATTCACCTCAACATCAGTCTGATAAGCTATATATTCACC

SPG3: GGTGAATATACTAATACTGCCTGGTAATGATGAGGTGAATATA, TATATTCACCTCATCATTACCAGGCAGTATTAGTATATTCACC

SPG4: GGTGAATATACTAATACTGAAAGGTAATGATGAGGTGAATATA, TATATTCACCTCATCATTACCTTTCAGTATTAGTATATTCACC

SPG5: GGTGAATATATGGGAGGGGAGAGGCAGCAAGCAGGTGAATATA, TATATTCACCTGCTTGCTGCCTCTCCСCTCCCATATATTCACC

SPG6: GGTGAATATATGGGAGGGGCACGGCAGCAAGCAGGTGAATATA TATATTCACCTGCTTGCTGCCGTGCCCCTCCCATATATTCACC

SPG7: GGTGAATATACATGCTGACCTCCCTCCTGCCCCAGGGTGAATATA TATATTCACCCTGGGGCAGGAGGGAGGTCAGCATGTATATTCACC
SPG8: GGTGAATATACATGCTGACCAAACTCCTGCCCCAGGGTGAATATA, TATATTCACCCTGGGGCAGGAGTTTGGTCAGCATGTATATTCACC

\section{Concatemerizing PCR}

PCR amplifications were performed in $50 \mu \mathrm{L}$ reactions at standard concentrations $[10 \mu \mathrm{L}$ of Bioline $5 \mathrm{xMyTaq}$ buffer (comprised of $5.0 \mathrm{mM}$ dNTPs, $15 \mathrm{mM} \mathrm{MgCl}$ ), $1.0 \mu \mathrm{L}$ Taq (Bioline, USA, Inc,. Randolph, MA), $0.2 \mu \mathrm{m}$ of each primer].The following cycling parameters: $94^{\circ} \mathrm{C}-2: 00 \mathrm{~min},\left(94^{\circ} \mathrm{C}-30 \mathrm{~s} 45^{\circ} \mathrm{C}-10 \mathrm{~s}, 72^{\circ} \mathrm{C}-30 \mathrm{~s}\right) \times 2$ cycles, $\left(94^{\circ} \mathrm{C}-30 \mathrm{~s}\right.$, $\left.40^{\circ} \mathrm{C}-30 \mathrm{~s}, 72^{\circ} \mathrm{C}-30 \mathrm{~s}\right) \times 29$ cycles, $72^{\circ} \mathrm{C}-1: 00$ minute, $4{ }^{\circ} \mathrm{C}$-or ever, were utilized. Following this, resulting amplicons were separated on a $1 \%$ agarose gel and desired sponge length was extracted. See "Supporting Information - Supplemental Protocol" for additional details.

\section{Vector cloning}

The band corresponding to desired sponge length was excised from the appropriate lane at $\sim 400 \mathrm{bp}$. Gel extractions were cloned into a pcr2.1-TOPOTA vector (Invitrogen). Concatemers were next excised from pcr2.1-TOPOTA and cloned into a mammalian expression vector (PCI, Promega, Madison, WI) using EcoR1 restriction sites which placed the sponge into the 3'UTR of the T7 promoter. Sponge orientation in PCI vector was determined by commercial sequencing (Functional Biosciences, Madison, WI). Sequencing primers for final constructs were: PCIF, TAATACGACTCACTATAGG; PCIR, AATAGCATCACAAATTTCAC.

\section{Results and discussion}

To achieve sponge production, $\sim 40 \mathrm{n} \mathrm{t}$ forward primers consisting of a simple linker (GGTGAATATA) flanking the 5' and 3' ends of an internal $\sim 20 \mathrm{nt}$ mi $\mathrm{R}$ sequence to be repeated across the sponge were designed and synthesized commercially. Reverse primers (the exact reverse complement of the forward primer) were also ordered, thereby allowing concatemerization of the sequence complementary to the mi R by the mechanism depicted in (Figure $2 \mathrm{~A})$. PCR amplifications were initially performed in $50 \mu \mathrm{L}$ reactions at standard concentrations. Following amplification, PCR products were separated by gel electrophoresis, and regions corresponding to $\sim 10$ repeats ( $\sim 400 \mathrm{bp}$ ) were manually excised (Figure $2 \mathrm{~B}$ ) then cloned into the PCR 2.1-TOPOTA (Invitrogen) vector (Figures 2C \& 3A). Next, to allow eukaryotic expression, sponges were removed from TOPO by the restriction digest of Eco RI sites immediately flanking both sides of the TOPO insertion, after which they were non-directionally sub cloned into a mammalian expression vector (PCI, Promega) linearized with ECORI (Figure 3B). Multiple colonies were isolated from resultant transformations and commercially sequenced (Functional Biosciences, Madison, WI). While expression vector sub cloning could alternatively be performed in a unidirectional manner following determination of orientation in PCR 2.1-TOPOTA by sequencing, this strategy allowed the simultaneous generation of both an expression vector producing a desired sponge and an identical expression vector producing the reverse complement of the sponge-an ideal transfection control. Importantly, Figure 2 demonstrates how a single round of PCR can be successfully employed to concatemerize single oligo nucleotide complements into markedly larger molecules tens of thousands of base pairs in length, from which any desired number of repeated miR target sites can be selected and utilized. Finally, we tested the versatility of our methodology by designing and attempting to amplify eight additional, unique sponges. Markedly, we found 
all eight primer sets robustly concatemerized under the conditions outlined here (Figure 4).

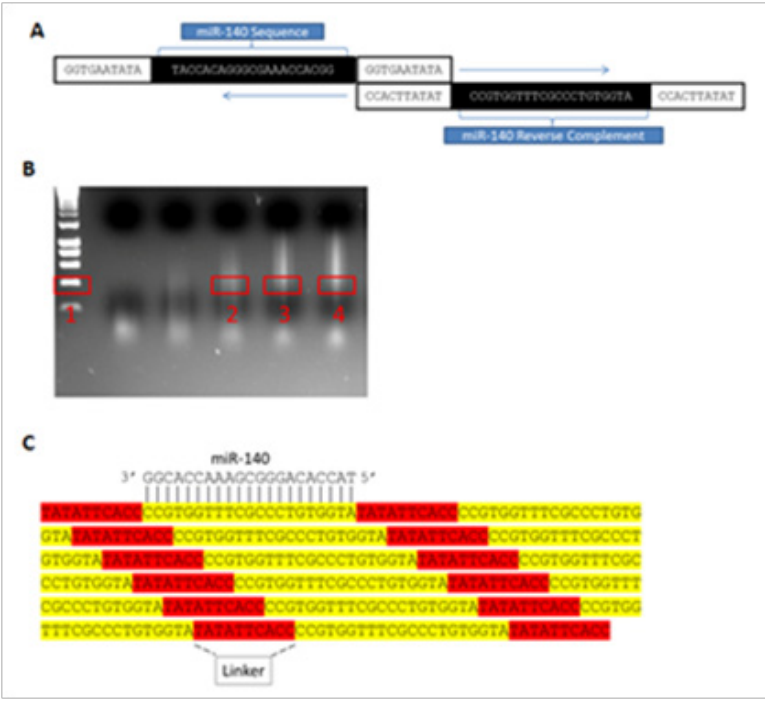

Figure 2 MicroRNA sponge production by concatemerizing PCR:

A. Complementary binding and elongation from concatemerizing primer linker sequences during $P C R$ amplification. miR Seq $R / C$, reverse complement of mature micro RNA.

B. Agarose gel separation of the products of three distinct concatemerizing PCRs. Box I, Hyper ladder I. Boxes 2-4, the regions ( 400bp) excised for sponge cloning. Distinct concatemerizations performed with the primers listed in Methods - Primer design.

C. MiR-140 sponge expression vector sequence confirming the presence of eleven miR-140 target sites (yellow) separated by linkers (red). The sequence is shown as it would be found in the expressed mRNA with putative miR-I 40 mi RNA binding indicated.

\section{A}

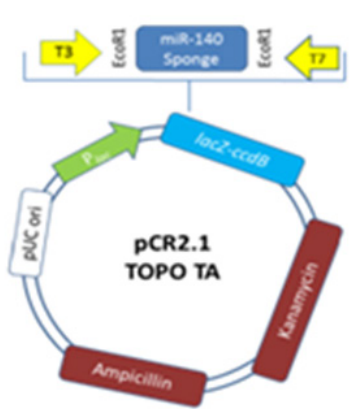

B

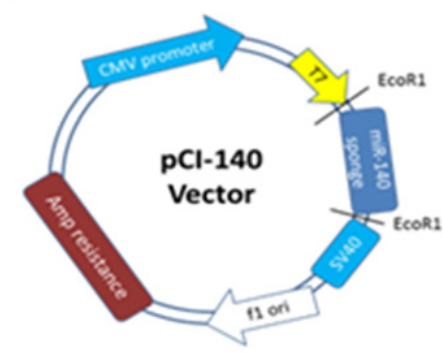

Figure 3 MiR-1 40 sponge clones.

A. PCR4-TOPO TA plasmid containing miR-I40 sponge between two EcoRI sites.

B. PCl mammalian expression vector containing miR-140 sponge.

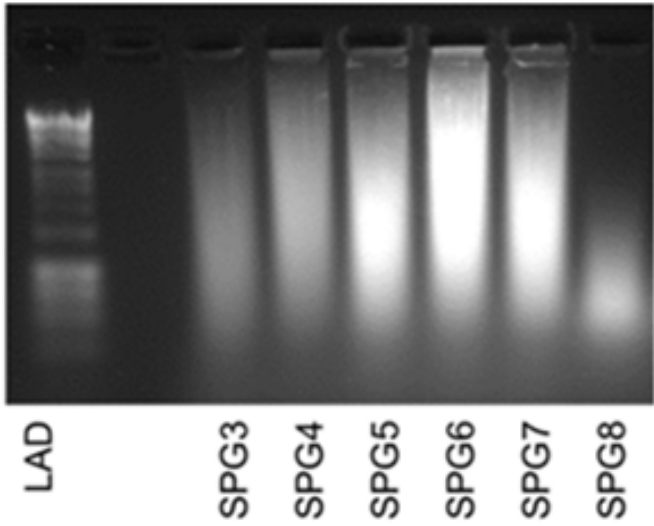

Figure 4 Additional concatemerizing PCRs: Agarose gel separation of the products of six distinct concatemerizing PCRs as in Figure 2B Concatemerizations were performed with the primers listed in Methods - Primer design.

\section{Conclusion}

In summary, as mi Rs clearly play prominent roles in disease, development and normal cellular metabolism, ${ }^{15}$ knocking down mi Rs has become an important area of research. As such, the discovery of several types of naturally occurring mi R sponges strongly supports the application of engineered mi R sponges in mi R inhibition. Naturally expressed circ RNA or circular RNA sponges (ciRS) can contain up to $70 \mathrm{mi} \mathrm{R}$ mismatched target sites capable of binding multiple mi $\mathrm{R}$ families ${ }^{16}$ much like newly discovered (ce RNAs) which also demonstrate endogenous mi R sponge-like activity. ${ }^{17} \mathrm{Ce}$ RNAs allow non-coding RNAs, such as mi Rs, to communicate via competition for mRNAs, with ce RNA function depending on mi R concentration. Ce RNAs contain binding sites for various mi Rs allowing them to regulate multiple mi $\mathrm{R}$ families. ${ }^{18}$ In addition, several other classes of non-coding RNAs may also function as endogenous mi R sponges (e.g. 3' UTR RNAs and linc RNAs). ${ }^{19}$

Currently, sponges are synthesized by either commercial synthesis or T4 ligation reactions. While more cost effective than commercial synthesis, due to the linear nature of their reaction mechanism, T4 ligation reactions are notoriously low throughput and typically require large amounts of starting material to produce relatively low product yields. ${ }^{14}$ In contrast, the method of sponge production we describe here represents a highly efficient, enzymatic reaction utilizing Taq polymerase to readily generate potent $\mathrm{mi} \mathrm{R}$ inhibitors that mimic endogenous sponges in a shorter time frame and at a substantially lower cost than existing production methods. Importantly, sponges can be constructed with repeats of any short sequence and designed to be perfect matches to mi Rs or instead to contain mismatches, or a central bulge (Figure 5), which has been suggested to be more effective at inhibiting miR function than perfectly complementary sponges. ${ }^{11}$ In addition, while we developed this PCR methodology to concatemerize miR target sites, we suggest this strategy could also be successfully utilized for other research purposes such as the concatemerization of transcription factor recruitment sites in expression constructs.

\section{>hsa-miR-140-3p MIMAT0004597 Original: TACCACAGGGTAGAACCACGG Altered: TACCACAGGGCGAAACCACGG}

Figure 5 MiR-I40 primer design: Original mature miR-I40 sequence with seed in red.Altered miR-140 sequence with central mismatch ("bulge) indicated by highlight. 


\section{Acknowledgements}

This work was facilitated by the University of South Alabama Department of Arts \& Sciences, the South Alabama University Committee on Undergraduate Research (UCUR) and the Genetics Department at the University of Iowa. Funding for this work was provided in part by NSF CAREER grant 1350064 (GB) awarded by Division of Molecular and Cellular Biosciences, with co-funding provided by the NSF EPSCoR program and the Abraham A. Mitchell Cancer Research Fund (GB).

\section{Conflict of interest}

The author declares no conflict of interest.

\section{References}

1. Wahid F, Khan T, Kim YY. MicroRNA and diseases: Therapeutic potential as new generation of drugs. Biochimie. 2014;104:12-26.

2. Borchert GM, Lanier W, Davidson BL. RNA polymerase III transcribes human micro RNAs. Nature structural \& molecular biology. 2006;13:1097-1101.

3. Martens Uzunova ES, Olvedy M, Jenster G. Beyond micro RNA-novel RNAs derived from small non-coding RNA and their implication in cancer. Cancer letters. 2013;340(2):201-211.

4. Hayes J, Peruzzi PP, Lawler S. Micro RNAs in cancer: biomarkers, functions and therapy. Trends in molecular medicine. 2014;20(8):460469.

5. Kim VN. MicroRNA biogenesis: coordinated cropping and dicing. Nature reviews Molecular cell biology. 2005;6(5):376-385.

6. Uchino K, Ochiya T, Takeshita F. RNAi therapeutics and applications of micro RNAs in cancer treatment. Jpn J Clin Oncol. 2013;43(6):596-607.

7. Iuliano R, Vismara MF, Dattilo V, et al. The role of micro RNAs in cancer susceptibility. Biomed Res Int. 2013;2013:591931.
8. Cheng Q, Yi B, Wang A, et al. Exploring and exploiting the fundamental role of micro RNAs in tumor pathogenesis. OncoTargets Ther. 2013;6:1675-1684.

9. Profumo V, Gandellini P. MicroRNAs: cobblestones on the road to cancer metastasis. Crit Rev Oncog. 2013;18(4):341-355.

10. Krutzfeldt J, Rajewsky N, Braich R, et al. Silencing of micro RNAs in vivo with 'antagomirs'. Nature. 2005;438(7068):685-689.

11. Ebert MS, Sharp PA. MicroRNA sponges: progress and possibilities. RNA. 2010;16(11):2043-2050.

12. Thomson DW, Bracken CP, Szubert JM, et al. On measuring mi RNAs after transient transfection of mimics or antisense inhibitors. PLoS One. 2013;8(1):e55214.

13. Stenvang J, Petri A, Lindow M, et al. Inhibition of micro RNA function by antimiR oligonucleotides. Silence. 2012;3(1):1.

14. Kluiver J, Slezak-Prochazka I, Smigielska-Czepiel K, et al. Generation of mi RNA sponge constructs. Methods. 2012;58(2):113-117.

15. Jiang Q, Wang Y, Hao Y, et al. miR2 Disease: a manually curated database for micro RNA deregulation in human disease. Nucleic Acids Res. 2009;37(Database issue):D98-D104.

16. Hansen TB, Jensen TI, Clausen BH, et al. Natural RNA circles function as efficient micro RNA sponges. Nature. 2013;495(7441):384-388.

17. Tay Y, Kats L, Salmena L, et al. Coding-independent regulation of the tumor suppressor PTEN by competing endogenous mRNAs. Cell. 2011;147(2):344-357.

18. Salmena L, Poliseno L, Tay Y, et al. A ceRNA hypothesis: the Rosetta Stone of a hidden RNA language? Cell. 2011;146(3):353-358.

19. Ebert MS, Sharp PA. merging roles for natural micro RNA sponges. Curr Biol. 2010;20(19):R858-R861. 